\title{
A comparison of the familiality of chronic depression in recurrent early-onset depression pedigrees using different definitions of chronicity
}

\author{
Francis M. Mondimore ${ }^{\mathrm{a}, *}$, Peter P. Zandi ${ }^{\mathrm{b}}$, Dean F. MacKinnon ${ }^{\mathrm{a}}$, Melvin G. McInnis ${ }^{\mathrm{c}}$, \\ Erin B. Miller ${ }^{a}$, Barbara Schweizer ${ }^{a}$, Raymond P. Crowe ${ }^{d}$, William A. Scheftner ${ }^{\mathrm{e}}$, \\ Myrna M. Weissman ${ }^{\mathrm{f}}$, Douglas F. Levinson ${ }^{\mathrm{g}}$, \\ J. Raymond DePaulo Jr. ${ }^{\text {a }}$, James B. Potash ${ }^{\text {a }}$ \\ Department of Psychiatry and Behavioral Sciences, School of Medicine, Johns Hopkins University, Baltimore, MD, USA \\ ${ }^{\mathrm{b}}$ Department of Mental Health, Bloomberg School of Public Health, Johns Hopkins University, Baltimore, MD, USA \\ ${ }^{\mathrm{c}}$ Department of Psychiatry, University of Michigan Medical School, Ann Arbor, Michigan, USA \\ ${ }^{\mathrm{d}}$ Department of Psychiatry and Mental Health Clinical Research Center, University of Iowa Carver College of Medicine, Iowa City, Iowa, USA \\ ' Department of Psychiatry, Rush-Presbyterian Medical Center, Chicago, Illinois, USA \\ ${ }^{\mathrm{f}}$ Department of Psychiatry, College of Physicians and Surgeons, Columbia University, New York; the New York State Psychiatric Institute, \\ New York, New York, USA \\ ${ }^{g}$ Department of Psychiatry, Stanford University School of Medicine, Palo Alto, California, USA
}

Received 29 August 2006; received in revised form 5 October 2006; accepted 13 October 2006 Available online 28 November 2006

\begin{abstract}
Background: The study of chronicity in the course of major depression has been complicated by varying definitions of this illness feature. Because familial clustering is one component of diagnostic validity we compared family clustering of chronicity as defined in the DSM-IV to that of chronicity determined by an assessment of lifetime course of depressive illness.

Methods: In 1750 affected subjects from 652 families recruited for a genetic study of recurrent, early-onset depression, we applied several definitions of chronicity. Odds ratios were determined for the likelihood of chronicity in a proband predicting chronicity in an affected relative.

Results: There was greater family clustering of chronicity as determined by assessment of lifetime course $(\mathrm{OR}=2.54)$ than by DSM-IV defined chronic major depressive episode (MDE) $(\mathrm{OR}=1.93)$ or dysthymic disorder $(\mathrm{OR}=1.76)$. In families with probands who had preadolescent onset of MDD, familiality was increased by all definitions, with a much larger increase observed for chronicity by lifetime course (ORs were 6.14 for lifetime chronicity, 2.43 for chronic MDE, and 3.42 for comorbid dysthymic disorder). Agreement between these definitions of chronicity was only fair.

Limitations: The data used to determine chronicity were collected retrospectively and not blindly to relatives' status, and assessment of lifetime course was based on global clinical impressions gathered during a semi-structured diagnostic interview. Also, it can be difficult to determine whether individuals with recurrent major depressive episodes who frequently experience long periods of low grade depressive symptoms meet the strict timing requirements of DSM-IV dysthymic disorder.
\end{abstract}

\footnotetext{
* Corresponding author. Johns Hopkins Hospital, Meyer 3-141, 600 North Wolfe Street, Baltimore, MD, 21287 , USA. Tel.: +1 410 614 1730; fax: +14109550152 .

E-mail address: fmondimo@jhmi.edu (F.M. Mondimore).
} 
Conclusions: An assessment of lifetime symptom course identifies a more familial, and thus possibly a more valid, type of chronic depression than the current DSM-IV categories which are defined in terms of particular cross-sectional features of illness.

(C) 2006 Elsevier B.V. All rights reserved.

Keywords: Depression; Chronic; Familial aggregation; Diagnosis

\section{Introduction}

Chronicity of depressive symptoms develops in approximately $25 \%$ of patients with major depressive disorder (MDD) and has been recognized as an important clinical feature of this illness (Judd et al., 1998). These patients suffer more morbidity and impairment than patients with non-chronic depressive illness and have been found to have increased rates of substance abuse and anxiety disorders (Crown et al., 2002) of attempted suicide (Klein et al., 2000), and substantial psychosocial and socioeconomic disability (Kennedy and Paykel, 2004).

The study of chronic depression has been complicated by varying definitions. DSM-IV categorizes chronic depression in three ways (American Psychiatric Association, 1994). (1) The specifier "chronic" may be applied to MDD if the current or most recent major depressive episode (MDE) has persisted for at least two years. (2) "Incomplete inter-episode recovery" is coded if, between the two latest episodes, symptoms no longer meet full diagnostic criteria but residual symptoms persist. (3) Dysthymic disorder is characterized by two or more years of depressive symptoms that do not meet MDE criteria, if there was no MDE within 6 months of the onset of the period.

Two other categories have been proposed that are not included in DSM-IV: The term "double depression" has been used to describe patients who have an MDE that follows two or more years of "minor depression" or dysthymic disorder (Keller and Lavori, 1984; Keller et al., 1997), and "depressive personality disorder," part of the DSM-IV appendix of "criteria sets...provided for further study," is defined in terms of "a pervasive pattern of depressive cognitions and behaviors" beginning in early adulthood.

Data are generally lacking to support the usefulness of these definitions for the identification of distinct subgroups of patients. One study comparing patients on a broad range of demographic, psychosocial and clinical variables found few differences between subjects in four sub-categories of chronic depression: a chronic MDE, recurrent MDD without full inter-episode recovery, MDE with dysthymic disorder, or chronic MDD with dysthymic disorder (McCullough et al., 2003).
One approach to assessing the validity of a diagnostic category is to determine whether it clusters in families (Feighner et al., 1972; Robins and Guze, 1970). Several studies have demonstrated familial aggregation of chronic depression. Relatives of 97 dysthymic probands were at increased risk of early-onset dysthymic disorder compared to relatives of probands with episodic MDD (Klein et al., 1995), and of depressive personality compared with relatives of non-depressed probands (Klein, 1999). We recently reported that chronicity as defined by lifetime assessment of course of illness is more common in relatives of MDD probands with chronic course compared with relatives of probands with non-chronic course (Mondimore et al., 2006).

Here, we extend our analysis to compare lifetime chronic course with two other chronicity categories as predictors of chronicity in families: history of a chronic MDE, and history of dysthymia in addition to MDD. We sought to determine which of these three definitions of chronicity is most predictive of familial clustering of chronicity, as an indication of the validity of the constructs. Note that our dataset did not permit us to study the concept of "double depression" described above, because it was not recorded whether an MDE was ever superimposed on a dysthymic period without remission from dysthymia before the MDE.

\section{Methods}

\subsection{Ascertainment}

The background and detailed methods of the six-site Genetics of Recurrent Early-onset Depression (GenRED I) project have been described elsewhere (Levinson et al., 2003). The GenRED I project primarily collected sibling pairs with recurrent early-onset MDD. For this report, data from 652 families were analyzed.

Each eligible family had a proband with recurrent early-onset MDD defined as sage 30 for probands. Recurrence was defined as at least two DSM-IV MDEs, except that subjects with a single MDE that lasted three years or more were also included. Probands were excluded if they had a diagnosis of schizophrenia, bipolar I or bipolar II disorders. Each proband had at least one sibling meeting the same criteria, but with 
onset at $\leq$ age 40 , and families were included if one or more siblings (but not the original proband) had a diagnosis of bipolar II disorder. Families were extended where possible to include other available affected firstdegree relatives meeting the same criteria as siblings. No affected individual was included in the study who had a parent or sibling considered likely (by interview or family history report) to have had a bipolar I disorder.

\subsection{Assessment}

After receiving an explanation of the study procedures, written informed consent was obtained from the subjects. Subjects were interviewed by trained research clinicians (masters level or higher) using the Diagnostic Interview for Genetic Studies (DIGS) (Nurnberger et al., 1994) version 3.0/GenRED, a semi-structured interview that assesses the severity and time course of depressive, manic and hypomanic symptoms over the subject's lifetime, as well as anxiety, psychotic, substance use, eating and antisocial disorders and medical history. In most cases, a Family Interview for Genetics Studies (FIGS) interview about the subject's history and symptoms was completed with a family informant (usually the other affected sibling), and psychiatric treatment records were often available.

DIGS interviews included an unstructured but detailed assessment of the subject's lifetime course of illness (periods of prodromal symptoms, active depression and residual symptoms) described in the interviewer's narrative report. The DIGS depression module includes ratings of the worst lifetime MDE and one other MDE, including specific symptoms, ages at onset and duration of the episodes, estimated number of lifetime episodes, and duration of longest episode. The DIGS dysthymia module then inquires whether the subject ever had "less severe episodes of depression that can go on for years at a time" and subjects who endorsed this item are asked about the symptoms, time course and exclusionary criteria for dysthymic disorder.
Two non-interviewing research clinicians independently reviewed all available data, assigned all applicable DSMIV diagnoses (with an age at onset and confidence level for each diagnosis), and made several additional ratings which included a global assessment of the lifetime course of MDD and the duration of the longest MDE. Course of MDD could be rated as "remitting" ("good remissions substantially longer than episodes"), "double or chronic" ("substantial mood symptoms most or all of the time"), "frequent/brief episodes ( $<3$ weeks) without prolonged remissions," or "other." Disagreements about diagnoses or other ratings were resolved by discussion or occasionally by review by a third clinician.

One limitation of the study is that the interviewer and diagnosticians for one family member were often not blind to the assessment of other family members. Because of the long duration of each interview and the fact that interviews and diagnostic reviews of family members were not usually completed consecutively, it seems unlikely that ratings of family members influenced each other, but the study procedure did not prevent this from occurring.

\subsection{Statistical analysis}

Inter-rater reliability for the chronicity assessment between first and second best estimate diagnostic reviewers was assessed by calculating the kappa statistic. Three definitions of chronicity were compared: 1) a history of a chronic MDE by DSM-IV criteria, 2) a bestestimate lifetime assessment of chronic course as discussed above and 3) lifetime diagnosis of dysthymic disorder in addition to major depression. We assessed the strength of the association between the different definitions of chronicity in subjects by calculating the kappa statistic, measuring whether the degree of agreement among definitions was greater than predicted by chance. Familial aggregation of chronicity was assessed using the generalized estimating equation (GEE). The GEE is an analysis of clustered data which uses logistic regression

Table 1

Comparison of probands and relatives: demographics and illness course

\begin{tabular}{lccrrr}
\hline & Probands $(N=652)$ & Relatives $(N=1098)$ & $\chi^{2 * *}$ & $p^{*}$ & All subjects $(N=1750)$ \\
\hline Gender & & & & & \\
$\quad$ Female & $550(84.4 \%)$ & $829(75.5 \%)$ & 19.20 & $<0.0001$ & $1379(78.8 \%)$ \\
$\quad$ Male & $102(15.6 \%)$ & $269(24.5 \%)$ & & $371(21.2 \%)$ \\
Chronicity as assessed by & & & & \\
$\quad$ Lifetime assessment & $228(35.0 \%)$ & $286(26.0 \%)$ & 15.70 & $<0.0001$ & $514(29.4 \%)$ \\
Chronic MDE & $163(25.0 \%)$ & $209(19.0 \%)$ & 8.70 & 0.003 & $372(21.3 \%)$ \\
Dysthymic disorder & $160(24.5 \%)$ & $199(18.1 \%)$ & 10.33 & 0.001 & $359(20.5 \%)$ \\
\hline
\end{tabular}

MDE: major depressive episode, ${ }^{*}$ two-sided $p$ values, $* * d f=1$. 
Table 2

Concordance of diagnoses in subjects with a chronicity diagnosis

\begin{tabular}{|c|c|c|c|}
\hline \multicolumn{4}{|c|}{ Diagnosed with chronicity by } \\
\hline & Life course & Chronic MDE & Dysthymic disorder \\
\hline \multicolumn{4}{|c|}{ Also diagnosed with chronicity by } \\
\hline $\begin{array}{l}\text { Life course } \\
(N=514)\end{array}$ & - & $\begin{array}{l}195 / 514 \\
(37.9 \%)\end{array}$ & $\begin{array}{l}241 / 514 \\
(46.9 \%)\end{array}$ \\
\hline $\begin{array}{l}\text { Chronic MDE } \\
(N=372)\end{array}$ & $\begin{array}{l}195 / 372 \\
(52.4 \%)\end{array}$ & - & $\begin{array}{l}97 / 372 \\
(26.0 \%)\end{array}$ \\
\hline $\begin{array}{l}\text { Dysthymic } \\
\text { disorder }\end{array}$ & $241 / 359$ & $97 / 359$ & - \\
\hline$(N=359)$ & $(67.1 \%)$ & $(27.0 \%)$ & \\
\hline
\end{tabular}

but also takes into account potential correlation between observations when multiple members of the same family are considered (Zeger and Liang, 1986). GEE was used to assess the degree to which chronicity in a proband predicted chronicity in the proband's affected first-degree relatives, while controlling for sex and for age at interview.

Additionally, GEE analyses were carried out with the sample stratified by pre-adolescent onset of major depressive disorder in probands (defined as prior to age thirteen years) versus post-adolescent onset (age 13 or older), because of our previous finding that a preadolescent onset of illness predicted stronger familial clustering of lifetime chronicity (Mondimore et al., 2006).

Demographic variables were contrasted in the three groupings. Student's $t$-test was used to compare probands and depressed relatives as well as chronically depressed and non-chronically depressed subjects for differences in age at time of interview, age at first MDE, longest MDE, total number of MDEs and number of years ill. Pearson's $\chi^{2}$ was used to test group differences in gender. The previously reported clinical correlates of chronicity (substance abuse, history of panic disorder, history of suicide attempt) (Mondimore et al., 2006) were assessed in subjects with and without chronic depression using Pearson's $\chi^{2}$.

\section{Results}

In 652 families, the proband had at least one fully assessed affected first-degree relative as defined above.

Table 3

Demographic and diagnostic comparisons using differing definitions of chronicity

\begin{tabular}{|c|c|c|c|c|}
\hline All subjects $N=1750$ & With chronicity & Without chronicity & & \\
\hline By lifetime assessment & $N=514$ & $N=1236$ & $\chi^{2}(d f=1)$ & $p^{*}$ \\
\hline Female & $406(79.0 \%)$ & $973(78.7 \%)$ & 0.01 & NS \\
\hline History of suicide attempt & $156(30.4 \%)$ & $204(16.5 \%)$ & 42.9 & $<0.0001$ \\
\hline Panic disorder & $152(29.6 \%)$ & $264(21.4 \%)$ & 13.5 & $<0.0001$ \\
\hline Substance abuse & $179(34.8 \%)$ & $335(27.1 \%)$ & $\begin{array}{l}10.4 \\
t(d f=1748)\end{array}$ & 0.001 \\
\hline Age of onset of MDD (years) & $18.3(\mathrm{SD}=8.5)$ & $20.4(\mathrm{SD}=8.6)$ & 4.58 & $<0.0001$ \\
\hline Number of MDEs & $7.0(\mathrm{SD}=11.5)$ & $6.4(\mathrm{SD}=0.30)$ & -1.3 & NS \\
\hline Longest MDE (days) & $1316.9(\mathrm{SD}=2029.2)$ & $468.3(\mathrm{SD}=538.0)$ & -13.6 & $<0.0001$ \\
\hline By chronic MDE & $N=372$ & $N=1378$ & $\chi^{2}(d f=1)$ & $p^{*}$ \\
\hline Female & $107(21.1 \%)$ & $260(21.4 \%)$ & 0.09 & NS \\
\hline History of suicide attempt & $104(28.0 \%)$ & $256(18.6 \%)$ & 15.71 & $<0.0001$ \\
\hline Panic disorder & $93(25.0 \%)$ & $323(23.44 \%)$ & 0.39 & NS \\
\hline Substance abuse & $122(32.8 \%)$ & $392(28.5 \%)$ & $\begin{array}{l}2.67 \\
t(d f=1748)\end{array}$ & NS \\
\hline Age of onset of MDD (years) & $18.8(\mathrm{SD}=9.3)$ & $20.0(\mathrm{SD}=8.4)$ & 2.37 & 0.02 \\
\hline Number of MDEs & $4.6(\mathrm{SD}=5.2)$ & $7.2(\mathrm{SD}=11.2)$ & 4.33 & $<0.0001$ \\
\hline Longest MDE (days) & $2193.4(\mathrm{SD}=2104.27)$ & $318.9(\mathrm{SD}=206.8)$ & -32.49 & $<0.0001$ \\
\hline Dysthymic disorder & $N=359$ & $N=1391$ & $\chi^{2}(d f=1)$ & $p^{*}$ \\
\hline Female & $289(80.5 \%)$ & $1090(78.4 \%)$ & 0.78 & NS \\
\hline History of suicide attempt & $96(26.7 \%)$ & $264(19.0 \%)$ & 10.48 & 0.001 \\
\hline Panic disorder & $98(27.3 \%)$ & $318(22.9 \%)$ & 3.10 & NS \\
\hline Substance abuse & $102(28.4 \%)$ & $412(29.6 \%)$ & $\begin{array}{l}0.20 \\
t(d f=1748)\end{array}$ & NS \\
\hline Age of onset of MDD (years) & $19.0(\mathrm{SD}=8.2)$ & $20.0(\mathrm{SD}=8.7)$ & 1.84 & NS \\
\hline Number of MDEs & $6.0(\mathrm{SD}=11.5)$ & $6.7(\mathrm{SD}=9.9)$ & 1.15 & NS \\
\hline Longest MDE (days) & $904.5(\mathrm{SD}=1470.2)$ & $669.7(\mathrm{SD}=1182.8)$ & -3.18 & 0.002 \\
\hline
\end{tabular}

* Two-sided $p$ values. 
Table 4

Odds ratios for chronic depression in affected relatives by chronicity status of probands

\begin{tabular}{|c|c|c|c|c|c|c|}
\hline & \multicolumn{6}{|c|}{ Proband chronicity status ${ }^{a}$} \\
\hline & Chronic & Non-chronic & Odds ratio ${ }^{\mathrm{b}}$ & SE & $z$ & $p$ \\
\hline \multicolumn{7}{|c|}{ Relatives with chronicity as assessed by } \\
\hline Lifetime assessment & $134 / 349(38.4 \%)$ & $152 / 749(20.3 \%)$ & 2.54 & 0.39 & 6.10 & $<0.0001$ \\
\hline Chronic MDE & $71 / 256(27.7 \%)$ & $138 / 842(16.4 \%)$ & 1.93 & 0.34 & 3.66 & $<0.0001$ \\
\hline Dysthymic disorder & $61 / 242(25.2 \%)$ & $138 / 856(16.1 \%)$ & 1.75 & 0.32 & 3.08 & 0.002 \\
\hline
\end{tabular}

${ }^{\text {a }}$ Proband and relative chronicity status determined using same definition in each analysis.

${ }^{\mathrm{b}}$ Odds ratio, $z$ and two-sided $p$ values calculated using logistic regression with generalized estimating equations to account for the residual correlation between multiple observations from the same family; all models controlled for age at interview and gender.

There were a total of 1098 fully assessed first-degree relatives: 901 siblings, 144 parents and 53 children. A summary of the characteristics of the probands compared to their relatives is given in Table 1. This is a slightly larger number of subjects than is reported in our previous report using this data set (Mondimore et al., 2006) because completed data on several additional families subsequently became available.

We found excellent agreement between first and second best-estimate diagnosticians for all chronicity determinations, with $89.7 \%$ agreement on chronicity by lifetime assessment (kappa $=0.76$, S.E. \pm 0.04$), 97.3 \%$ agreement for DSM-IV chronic MDE (kappa=0.93, S.E. \pm 0.03 ), and $94.6 \%$ agreement on the diagnosis of dysthymic disorder (kappa $=0.86$, S.E. \pm 0.03 .) Table 1 shows that the proportion of all MDE subjects who were diagnosed with depression chronicity by lifetime assessment (514/1750, 29.4\%) was higher than chronicity diagnosed by a chronic MDE (372/1705, 21.3\%) or with the additional diagnosis of dysthymic disorder (359/1750, 20.5\%). Table 2 shows the overlap between each pair of definitions. The best agreement was between lifetime assessment and dysthymia (kappa $=0.41$, S.E. \pm 0.02 ), with lower agreement between the lifetime assessment and chronic MDE definitions (kappa $=0.26$, S.E. \pm 0.02 ) and essentially no agreement between chronic MDE and the diagnosis of dysthymic disorder (kappa $=0.07$, S.E. \pm 0.02 ).

Because our previous analysis of this dataset showed that chronic subjects had an earlier age of onset, and higher rates of attempted suicide, panic disorder, and substance misuse disorders than episodic subjects, we also compared these variables across the differing definitions of chronicity (see Table 3 ). When chronic MDE and a diagnosis of dysthymic disorder were used to define chronicity, differences between panic disorder, substance misuse and age of onset in chronic versus non-chronic subjects were less pronounced.

The results of the primary analysis of familial aggregation of chronicity under varying definitions are shown in Table 4. Among probands with lifetime chronicity, $38.4 \%$ of relatives were assessed as having lifetime chronicity, compared to $20.3 \%$ of relatives of non-lifetime chronic probands $(\mathrm{OR}=2.54, z=6.10$, $p<0.0001)$. Among probands with a history of a chronic MDE, $27.7 \%$ of relatives had a chronic MDE, compared to $16.4 \%$ of relatives of non-chronic MDE probands $(\mathrm{OR}=1.93, z=3.66, p<0.0001)$. Among probands with dysthymic disorder, $25.2 \%$ had a period of dysthymia,

Table 5

Odds Ratios (OR) for chronic depression in affected relatives by chronicity status of probands stratified by pre-adolescent versus post-adolescent illness onset

\begin{tabular}{|c|c|c|c|c|c|c|c|c|c|c|c|c|}
\hline & \multicolumn{6}{|c|}{ Preadolescent onset probands $(n=129)$} & \multicolumn{6}{|c|}{ Post-adolescent onset probands $(n=523)$} \\
\hline & \multicolumn{6}{|l|}{ Proband status ${ }^{\mathrm{a}}$} & \multicolumn{6}{|l|}{ Proband status $^{\mathrm{a}}$} \\
\hline & Chronic & Non-chronic & $\mathrm{OR}^{\mathrm{b}}$ & SE & $z$ & $p$ & Chronic & Non-chronic & $\mathrm{OR}^{\mathrm{b}}$ & $\mathrm{SE}$ & $z$ & $p$ \\
\hline \multicolumn{13}{|c|}{ Relatives with chronicity as assessed by } \\
\hline $\begin{array}{l}\text { Lifetime } \\
\text { assessment }\end{array}$ & $42 / 85(49.4 \%)$ & $18 / 128(14.1 \%)$ & 6.14 & 2.09 & 5.33 & $<0.0001$ & $92 / 264(34.8 \%)$ & $134 / 621(20.0 \%)$ & 2.00 & 0.35 & 3.98 & $<0.0001$ \\
\hline Chronic MDE & $19 / 74(25.7 \%)$ & $17 / 139(12.2 \%)$ & 2.43 & 0.93 & 2.32 & 0.02 & $52 / 182(28.6 \%)$ & $121 / 703(17.2 \%)$ & 1.92 & 0.40 & 3.15 & 0.002 \\
\hline $\begin{array}{l}\text { Dysthymic } \\
\text { Disorder }\end{array}$ & $16 / 43(37.2 \%)$ & $25 / 170(14.7 \%)$ & 3.42 & 1.34 & 3.15 & 0.002 & $45 / 199(22.6 \%)$ & $113 / 686(16.5 \%)$ & 1.51 & 0.31 & 1.98 & 0.05 \\
\hline
\end{tabular}

\footnotetext{
a Proband and relative chronicity status determined using same definition in each analysis.

${ }^{\mathrm{b}}$ Odds ratio, $z$ and two-sided $p$ values calculated using logistic regression with generalized estimating equations to account for the residual correlation between multiple observations from the same family; all models controlled for age at interview and gender.
} 
compared to $16.1 \%$ of relatives of non-dysthymic probands $(\mathrm{OR}=1.76, z=3.08, p=0.002)$. We could not formally assess whether differences between the ORs for familial aggregation measured with each chronicity definition were statistically significant because the different definitions captured overlapping rather than independent groups of subjects.

Because our previous analysis of this dataset showed a striking increase in familial clustering when the data were stratified by pre-adolescent versus post-adolescent onset of major depressive disorder, we again tested for family clustering while limiting the analysis to families in which the probands had an onset of illness before age 13. We found an increased clustering of chronicity in these families using all three definitions of chronic depression, with the most robust increase seen when lifetime chronicity was used. These results are summarized in Table 5. The degree of familial aggregation under the lifetime chronicity definition was greater than under either of the DSM- IV diagnoses of chronic depressive conditions.

\section{Discussion}

In families ascertained for recurrent early-onset MDD, chronic depressive illness as defined by a global lifetime clinical assessment of chronicity shows greater familiality than chronicity defined by at least one DSM-IV chronic MDE or by an additional diagnosis of dysthymic disorder, a finding that is most pronounced in relatives of probands with pre-adolescent onset of MDD. Lifetime assessment of chronicity was most strongly predictive of substance misuse, panic disorder and earlier age of onset.

In 1970, Robins and Guze proposed a method for "achieving diagnostic validity in psychiatric illness" and suggested that "the finding of an increased prevalence of the same disorder among the close relatives of the original patients strongly indicates that one is dealing with a valid entity" (Robins and Guze, 1970). Our previous results and those of others suggest that "chronic depression" is a valid diagnostic entity by this criterion. If stronger family clustering indicates greater diagnostic validity, then the findings we present here suggest that an assessment of lifetime symptom course may be more important for the identification of the subset of patients with chronic depressive illness than diagnostic approaches which focus on particular episodes of illness and may fail to capture the long-term persistence of residual symptoms (Judd et al., 1998).

Our data are consistent with studies showing family clustering of chronic MDE and double depression.
Klein and colleagues reported that relatives of subjects with dysthymic disorder ( $77 \%$ of whom had a lifetime diagnosis of MDD) were more likely to suffer from dysthymia or major depressive disorder than relatives of controls (Klein et al., 1995). However, in another report (Klein et al., 2004) these authors did not find significant familiality of several definitions of chronic depression. In 1309 relatives of probands with dysthymic disorder $(n=95)$, chronic major depression $(n=65)$ or episodic major depression $(n=313)$, and 1306 relatives of probands with no mood disorder $(n=392)$, chronic major depression was not significantly more common in the relatives of probands with chronic MDD, and dysthymic disorder was not significantly more likely in relatives of dysthymic versus non-dysthymic probands. The division of chronicity into separate forms rather than viewing it as "a single broad condition" (McCullough et al., 2003) may have diluted this study's statistical power to detect family clustering.

Strengths of our study include the assessment of subjects by direct interview with a validated instrument (DIGS) usually supplemented by family reports and clinical records, the large sample size, and the excellent inter-rater reliability for all three definitions of chronicity. The study has several limitations. First, we did not have data on the "major depressive disorder with incomplete inter-episode recovery" category or "depressive personality." Second, because all data except for treatment records were retrospective, faulty recall of details of illness course could have produced an assessment error (Andrews et al., 1999). However, multiple sources of information increase the validity of the ratings. Third, the assessment of lifetime chronicity in the interview was based on the unstructured illness overview portion of the DIGS, not questions that systematically addressed lifetime course. However, the inter-rater reliability for the chronicity assessment between first and second bestestimate reviewers was excellent. Fourth, the DIGS dysthymia module is less detailed than the major depression and mania modules. Further, it is inherently difficult to evaluate the presence or absence of dysthymic disorder in individuals with recurrent MDD who also experience long periods of lower-grade depressive symptoms, particularly in a retrospective study (Judd et al., 1998). Lastly, the lifetime assessment of chronicity might hypothetically cluster more strongly than the DSMIV chronicity categories if it were based on a measurement procedure that had higher reliability rather than greater diagnostic validity. We found, however, that the kappa statistics did not significantly differ across the three measures, indicating similar levels of reliability for each. 
We would propose that research on chronic depression should consider an assessment of the lifetime course of illness based on all sources of information, and that this construct may be more valid and useful than categorizing subjects by the cross-sectional DSM-IV criteria for chronic MDE or comorbid dysthymic disorder. The use of a chronicity variable could inform future genetic studies of mood disorders. Further refinement of the definition of chronicity in depressive illness might increase the power of such studies.

\section{Acknowledgements}

Supported by a grant from The National Alliance for Research on Schizophrenia and Depression (Mondimore). Data collected for the National Institute of Mental Health Genetics of Recurrent Early-Onset Depression (GenRED) project, supported by NIMH grants 5R01MH059542 (Crowe), 5R01MH059552-04 (DePaulo), 5R01MH061686 (Levinson), 5R01MH059541 (Scheftner) and 5R01MH060912 (Weissman). We acknowledge the contributions of Dr. George S. Zubenko and Dr. Wendy N. Zubenko (supported by 5R01MH060866) and of the NIMH Center for Collaborative Genetic Studies on Mental Disorders.

\section{References}

American Psychiatric Association, 1994. Diagnostic and Statistical Manual of Mental Disorders, Fourth ed. American Psychiatric Association, Washington, DC.

Andrews, G., Anstey, K., Brodaty, H., Issakidis, C., Luscombe, G., 1999. Recall of depressive episode 25 years previously. Psychol. Med. 29, 787-791.

Crown, W.H., Finkelstein, S., Berndt, E.R., Ling, D., Poret, A.W., Rush, A.J., Russell, J.M., 2002. The impact of treatment-resistant depression on health care utilization and costs. J. Clin. Psychiatry 63, 963-971.

Feighner, J.P., Robins, E., Guze, S.B., Woodruff, R.A., Winokur, G., Munoz, R., 1972. Diagnostic criteria for use in psychiatric research. Arch. Gen. Psychiatry 26, 57-63.

Judd, L.L., Akiskal, H.S., Maser, J.D., Zeller, P.J., Endicott, J., Coryell, W., Paulus, M.P., Kunovac, J.L., Leon, A.C., Mueller, T.I., Rice, J.A., Keller, M.B., 1998. A prospective 12-year study of subsyndromal and syndromal depressive symptoms in unipolar major depressive disorders. Arch. Gen. Psychiatry 55, 694-700.
Keller, M.B., Lavori, P.W., 1984. Double depression, major depression, and dysthymia: distinct entities or different phases of a single disorder? Psychopharmacol. Bull. 20, 399-402.

Keller, M.B., Hirschfeld, R.M., Hanks, D., 1997. Double depression: a distinctive subtype of unipolar depression. J. Affect. Disord. 45, 65-73.

Kennedy, N., Paykel, E.S., 2004. Residual symptoms at remission from depression: impact on long-term outcome. J. Affect. Disord. 80, 135-144.

Klein, D.N., 1999. Depressive personality in the relatives of outpatients with dysthymic disorder and episodic major depressive disorder and normal controls. J. Affect. Disord. 55, 19-27.

Klein, D.N., Riso, L.P., Donaldson, S.K., Schwartz, J.E., Anderson, R.L., Ouimette, P.C., Lizardi, H., Aronson, T.A., 1995. Family study of early-onset dysthymia. Mood and personality disorders in relatives of outpatients with dysthymia and episodic major depression and normal controls. Arch. Gen. Psychiatry 52, 487-496.

Klein, D.N., Schwartz, J.E., Rose, S., Leader, J.B., 2000. Five-year course and outcome of dysthymic disorder: a prospective, naturalistic follow-up study. Am. J. Psychiatry 157, 931-939.

Klein, D.N., Shankman, S.A., Lewinsohn, P.M., Rohde, P., Seeley, J.R., 2004. Family study of chronic depression in a community sample of young adults. Am. J. Psychiatry 161, 646-653.

Levinson, D.F., Zubenko, G.S., Crowe, R.R., DePaulo, R.J., Scheftner, W.S., Weissman, M.M., Holmans, P., Zubenko, W.N., Boutelle, S., Murphy-Eberenz, K., MacKinnon, D., McInnis, M.G., Marta, D.H., Adams, P., Sassoon, S., Knowles, J.A., Thomas, J., Chellis, J., 2003. Genetics of recurrent early-onset depression (GenRED): design and preliminary clinical characteristics of a repository sample for genetic linkage studies. Am. J. Med. Genet. 119B, 118-130.

McCullough Jr., J.P., Klein, D.N., Borian, F.E., Howland, R.H., Riso, L.P., Keller, M.B., Banks, P.L., 2003. Group comparisons of DSM-IV subtypes of chronic depression: validity of the distinctions, part 2. J. Abnorm. Psychology 112, 614-622.

Mondimore, F.M., Zandi, P., MacKinnon, D., McInnis, M., Miller, E., Crowe, R., Scheftner, W., Marta, D.H., Weissman, M.M., Levinson, D.F., Murphy-Eberenz, K., DePaulo Jr., J.R., Potash, J.B., 2006. Familial aggregation of illness chronicity in recurrent, early-onset depression pedigrees. Am. J. Psychiatry 163, 1554-1560.

Nurnberger Jr., J.I., Blehar, M.C., Kaufmann, C.A., York-Cooler, C., Simpson, S.G., Harkavy-Friedman, J., Severe, J.B., Malaspina, D., Reich, T., 1994. Diagnostic interview for genetic studies. Rationale, unique features, and training. NIMH genetics initiative. Arch. Gen. Psychiatry 51, 849-859.

Robins, E., Guze, S.B., 1970. Establishment of diagnostic validity in psychiatric illness: its application to schizophrenia. Am. J. Psychiatry $126,983-987$.

Zeger, S.L., Liang, K.Y., 1986. Longitudinal data analysis for discrete and continuous outcomes. Biometrics 42, 121-130. 